\title{
Perception of Unfamiliar English Phonemes by Native Mandarin Speakers
}

\author{
Gabriela Holko ${ }^{1}$, Scott James Perry ${ }^{1}$, Matthew C. Kelley ${ }^{1}$, Benjamin V. Tucker ${ }^{1}$ \\ ${ }^{1}$ Department Linguistics, University of Alberta
}

\begin{abstract}
In second language acquisition, speech sounds, or phonemes, not present in a learner's native language often pose an extra challenge for speech production. When hearing one of these unfamiliar phonemes, the learner either maps it to a similar native phoneme, perceives it as a completely foreign sound, or does not perceive it as speech at all. In the first case, the learner is unable to perceive a difference between the unfamiliar phoneme and the native phoneme to which it is mapped. This mapping difficulty potentially creates problems for the learner during word recognition. The present research investigated the extent to which English phonemes absent from the Mandarin phonological inventory impact processing of native Mandarin speakers in an auditory lexical decision task. Results of this research will expand the understanding of second language perception, especially within the context of auditory lexical

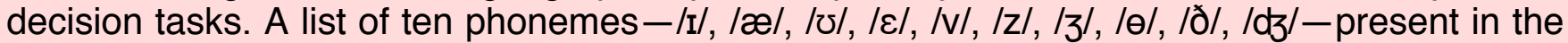
English phonological inventory but absent from that of Mandarin were identified as unfamiliar to native Mandarin speakers. Data from the Massive Auditory Lexical Decision (MALD) database, in which participants decided whether recorded utterances were English words or made-up words, were utilized. The effects of the proportion of unfamiliar phonemes, proportion of unfamiliar vowels, and proportion of unfamiliar consonants on reaction time, representative of processing difficulty, were then calculated using statistical techniques. It was found that the proportion of all unfamiliar phonemes in an utterance had no significant effect on the reaction time of the native Mandarin speakers. However, when the list of unfamiliar phonemes was divided into vowels and consonants, a greater proportion of unfamiliar vowels was noticed to increase reaction time, while a greater proportion of unfamiliar consonants was found to decrease reaction time. Further research in this area is required to determine a concrete explanation for these results. Interestingly, when the same analysis was performed on the data of native English speakers, similar results were observed. This may reflect a common language processing mechanism in second language learners and native speakers.
\end{abstract}

Key words:

auditory lexical decision, spoken word recognition, second language perception, L2, L2 perception, L1 Mandarin, L2 English

Cite as: Holko G., Perry, S.J., Kelley M.C., and Tucker B.V. 2019. Perception of unfamiliar English phonemes by native Mandarin speakers. Alberta Academic Review, Vol 2 (2) 37-38, WISEST Special Issue (not peer-reviewed), DOI 10.29173/aar46. 
Holko et al., 2019

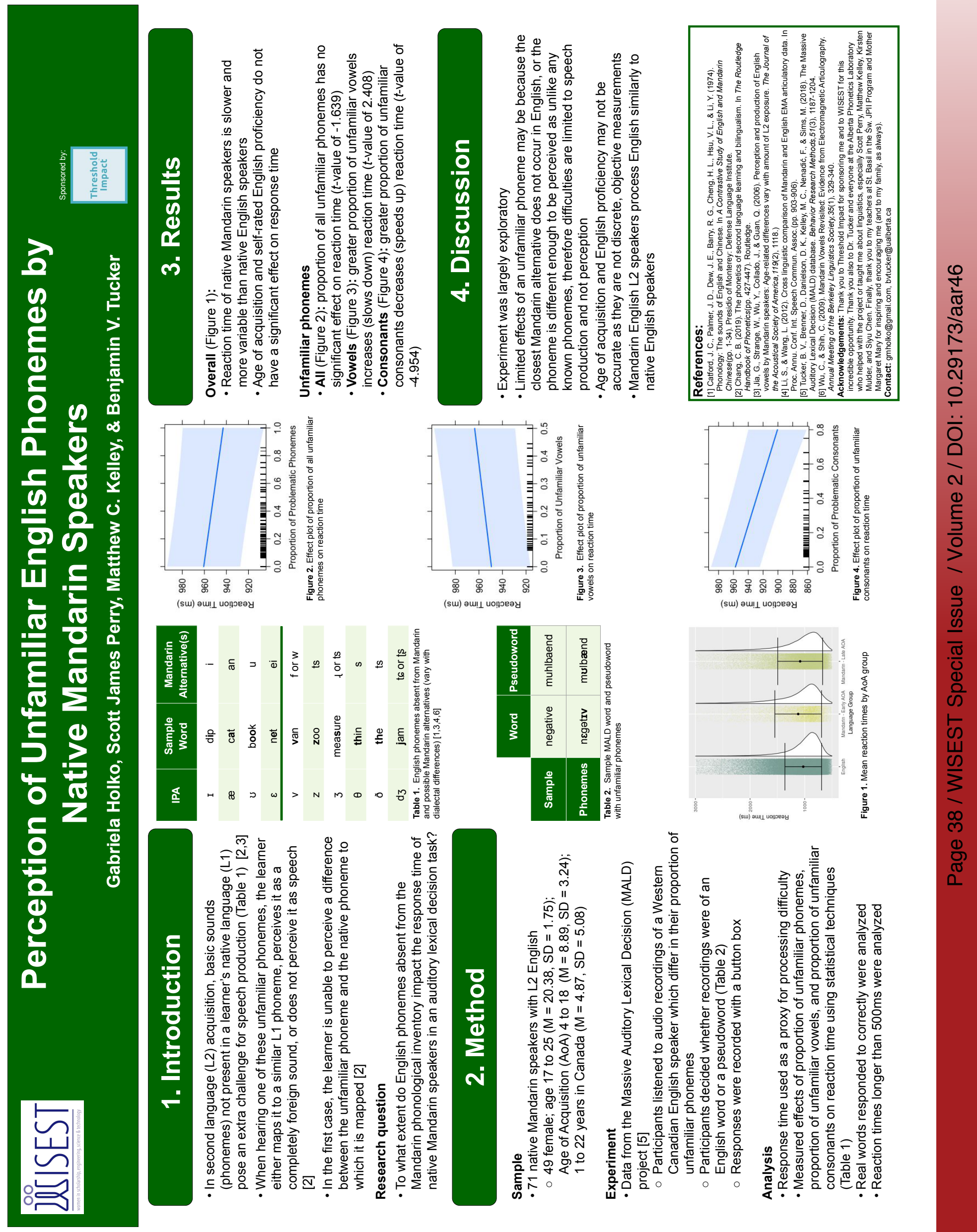

